\title{
Behaviour change in overweight and obese pregnancy: a decision tree to support the development of antenatal lifestyle interventions
}

\author{
Kate M Ainscough ${ }^{1}$, Karen L Lindsay ${ }^{1}$, Elizabeth J O'Sullivan ${ }^{1}$, Eileen R Gibney ${ }^{2}$ and \\ Fionnuala M McAuliffe ${ }^{1, *}$ \\ 'UCD Perinatal Research Centre, School of Medicine, University College Dublin, National Maternity Hospital, \\ Dublin, Republic of Ireland: ${ }^{2}$ UCD Institute of Food and Health, School of Agriculture and Food Science, Dublin, \\ Republic of Ireland
}

Submitted 14 November 2016: Final revision received 31 March 2017: Accepted 17 May 2017: First published online 10 July 2017

\begin{abstract}
Objective: Antenatal healthy lifestyle interventions are frequently implemented in overweight and obese pregnancy, yet there is inconsistent reporting of the behaviour-change methods and behavioural outcomes. This limits our understanding of how and why such interventions were successful or not.

Design: The current paper discusses the application of behaviour-change theories and techniques within complex lifestyle interventions in overweight and obese pregnancy. The authors propose a decision tree to help guide researchers through intervention design, implementation and evaluation. The implications for adopting behaviourchange theories and techniques, and using appropriate guidance when constructing and evaluating interventions in research and clinical practice are also discussed.

Conclusions: To enhance the evidence base for successful behaviour-change interventions during pregnancy, adoption of behaviour-change theories and techniques, and use of published guidelines when designing lifestyle interventions are necessary. The proposed decision tree may be a useful guide for researchers working to develop effective behaviour-change interventions in clinical settings. This guide directs researchers towards key literature sources that will be important in each stage of study development.
\end{abstract}

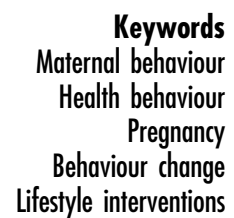

The growing prevalence of overweight and obesity among expectant mothers has a complex aetiology ${ }^{(1)}$. Although it may be driven by a diet rich in foods that are high in energy, fat and sugar, and poor engagement in physical activity during pregnancy ${ }^{(1)}$, contextual factors such as the physical environment and the mother's social network and emotional well-being likely also contribute. In pregnant women with increased adiposity, these behaviours have been associated with increased risk of excess gestational weight gain (GWG), heightening the incidence of adverse pregnancy outcomes and long-term health consequences for the mother and her offspring ${ }^{(1)}$. Improving diet and physical activity behaviours during pregnancy can improve short-term pregnancy outcomes as well as longterm maternal and offspring health ${ }^{(2)}$.

Women often perceive a number of barriers and facilitators to changing health behaviours during pregnancy ${ }^{(3)}$. However, compared with normal-weight women, women with a higher BMI may perceive greater barriers to adopting lifestyle changes, which can outweigh facilitating influences ${ }^{(3)}$. Thus, effective antenatal lifestyle interventions are urgently required to assist overweight and obese pregnant women to implement healthy behaviour changes to improve health outcomes.

\section{Current issue: problems interpreting behaviour- change interventions during pregnancy}

Clinical outcomes are often the major focus of interventions, which can mean that changes in the dietary and physical activity behaviours targeted to achieve the primary clinical outcomes are sometimes overlooked ${ }^{(4-6)}$. This results in unanswered questions such as: what behaviour-change theories and techniques were used; were the desired behaviours and respective influencing factors adequately targeted; and did the target behaviours improve following the intervention? Intensive lifestyle interventions have successfully changed lifestyle behaviours in overweight and 
obese pregnant women ${ }^{(7,8)}$. However, financial, time and labour constraints limit the feasibility of intensive interventions in clinical practice ${ }^{(9)}$. Thus, determining the most successful and cost-effective components of interventions is an important aspect of translational research in this field ${ }^{(9)}$.

Researchers should be cognisant of the specific behaviour warranting change, the factors that influence the behaviour, and ways to systematically design, implement and evaluate interventions that can change behaviour ${ }^{(10)}$. This systematic approach is achievable using appropriate evidence, tools and guidance, and facilitates the translation of behaviourchange research into clinical practice. The current paper proposes a decision tree to assist the design, implementation and evaluation of behaviour-change interventions among overweight and obese pregnant women.

\section{Understanding behaviours and behaviour change: addressing contextual influences among pregnant women}

Formative qualitative research has identified obese women's perceptions of barriers and facilitators to changing diet and physical activity during pregnancy, which may be categorised as: physiological; emotional; environmental; cognitive; and interpersonal factors ${ }^{(3)}$. Perceived barriers often outnumber facilitators among women, resulting in reduced self-efficacy to initiate change $^{(3)}$. Additionally, barriers and facilitators may differ depending on a woman's environment. Thus, identification of contextually relevant barriers to behaviour change is a critical step in the design of effective interventions.

Some trials have performed pilot or feasibility testing to gain participant feedback on planned interventions ${ }^{(11-14)}$. Qualitative data and pilot testing during developmental stages of an intervention can refine methods for large-scale trials, increasing the likelihood of success. Two recent qualitative studies have explored pregnant women's thoughts, ideas and perceptions on the design of trials to encourage healthy $\mathrm{GWG}^{(13,14)}$. In the first study ${ }^{(13)}$, to aid the development of an ongoing lifestyle intervention among overweight and obese African-American pregnant women, participant interviews based on the social ecological model $^{(15)}$ were used to assess women's perceptions of a healthy GWG and any barriers or enablers they perceived to healthy eating and physical activity ${ }^{(13)}$. In the second study among low-income, overweight and obese women, focus groups were conducted to inform the design of an ongoing stress-reduction intervention to target the root cause of lifestyle behaviours which influence $\mathrm{GWG}^{(14)}$.

\section{Assessing behaviour change: measuring how an intervention leads to behaviour change}

To assess whether an intervention changes behaviour, we must measure any changes in target behaviours as well as behavioural constructs. In previous randomised controlled trials among overweight and obese pregnant women, subjective and/or objective measures of dietary and physical activity behaviours targeted by interventions to improve pregnancy outcomes have not always been reported $^{(4-6,16)}$. Trials aiming to reduce GWG via a diet and exercise intervention ${ }^{(4)}$ and via a dietary intervention alone $^{(6)}$ did not report changes in diet and physical activity - the behaviours directly influencing GWG. The former study employed behaviour-change theory and techniques $^{(4)}$. Similarly, an intense dietary-behavioural intervention aiming to reduce GWG, infant birth weight and the incidence of gestational diabetes, which also employed behaviour-change techniques, reported only the frequency of certain foods consumed the day before the first and final study visits during pregnancy ${ }^{(5)}$. Although this provides some knowledge of the participants' diet, it is not sufficient to provide a reliable estimate of changes in energy and nutrient intakes which may affect GWG. Some trials measure adherence to intervention recommendations for these behaviours, but not the overall improvement, if any, in dietary or nutrient intakes or activity levels ${ }^{(17-19)}$. The tide may be shifting, however, as recently published antenatal intervention trials ${ }^{(20,21)}$ have reported changes in dietary and physical activity behaviours and clinical outcomes among overweight and obese pregnant women, namely the incidence of infants born large-for-gestational-age ${ }^{(20)}$ and gestational diabetes $^{(21)}$. Contemporary trials have also measured changes in psychological behavioural constructs, such as self-efficacy, well-being and depression ${ }^{(18,21)}$. Researchers are also now designing patient-informed interventions which have a strong psychological focus ${ }^{(14)}$. Measuring alterations in psychological behavioural constructs, or performing mediation analyses, might provide insight to the underlying mechanisms by which an intervention may change behaviour. For example, a recent study protocol details how it will statistically measure the mediating effect of social support, self-efficacy, self-regulation and motivation - as measured by individual questionnaires among women enrolled in a dietary and physical activity intervention to manage $\mathrm{GWG}^{(22)}$.

The use of implicit/explicit theory and techniques to change diet and/or physical activity behaviours within antenatal interventions has varied. Although it is possible that behaviour-change theories and techniques are used in all published lifestyle interventions, reporting of the same is insufficiently detailed, as some trials have described neither ${ }^{(6,7,16)}$ or described techniques only ${ }^{(23)}$. More recently published and ongoing trials have been more detailed in their reporting; detailing theory and defined behaviour-change techniques ${ }^{(21,24)}$ to successfully change diet and physical activity in overweight and obese pregnant women. Furthermore, intervention mapping protocols, which use an iterative path to map out an intervention and its hypothesised effect on behaviour 
change, could be of benefit when planning antenatal interventions $^{(22,25)}$. The recent detailed reporting of specific behaviour-change theories, techniques and protocols from behavioural science in antenatal interventions is promising ${ }^{(21,24)}$, encouraging further research.

\section{Where can we go from here?}

To improve the quality of research and create a better evidence base for clinical practice, we recommend researchers universally adopt systematic methods for constructing behaviour-change interventions during pregnancy; incorporating behaviour-change theories and techniques along with formative qualitative research and trial piloting. Recognising the need for systematic reporting of behaviour-change interventions across all disciplines, the Medical Research Council (MRC) ${ }^{(26)}$ and the National Institute for Health and Clinical Excellence $(\mathrm{NICE})^{(27)}$ in the UK have developed generic protocol guidelines for formulating complex behaviour-change interventions. Another approach to behavioural intervention design is the Multiphase Optimisation Strategy $\left(\right.$ MOST) ${ }^{(28)}$. The MOST approach involves a three-phase development structure in which behavioural interventions are optimised prior to commencement of randomised controlled trials to maximise the suitability of the intervention and its impact within the target population ${ }^{(28)}$. Interventions are optimised using any experimental design, but favourably a Sequential Multiple Assignment Randomised Trial (SMART). This differs from classic pilot interventions in that several treatments are tested to identify and select one with optimal components for implementation in the randomised controlled trial phase $^{(28)}$.

Additionally, the afore-mentioned intervention mapping, involving logic models, can be used to systematically construct interventions ${ }^{(22,25)}$. Intervention mapping protocols and programme impact pathways (PIP) allow researchers to determine the impact of behaviour-change components within interventions on outcomes ${ }^{(22,29,30)}$. These pathways and protocols can take contextual factors as well as behaviour-change theory into account. Thus, performing intervention mapping or PIP analysis can help researchers establish what behaviour-change components worked, and why and how they worked ${ }^{(22,29)}$.

Regarding the approaches discussed above, researchers with limited knowledge and experience of behaviour change may benefit from structured support when designing antenatal interventions.

\section{Structured support for developing antenatal behaviour-change interventions}

The decision tree proposed in the present paper (Fig. 1) is a step-by-step guide through the design, implementation and evaluation stages of antenatal dietary and physical activity interventions. This framework was developed using information drawn from scientific and grey literature to collate information about the use of behaviour-change theories and techniques and developing behavioural interventions. The decision tree also highlights intervention mapping techniques and demonstrates how these can be used in intervention research. Recent systematic reviews of interventions incorporating $\operatorname{diet}^{(31)}$ or diet and physical activity ${ }^{(32-40)}$ to improve GWG and the adverse pregnancy outcomes associated with maternal obesity were examined. Additionally, recent Cochrane reviews of dietary and physical activity ${ }^{(41)}$ and dietary-only ${ }^{(42)}$ antenatal lifestyle interventions to reduce the incidence of gestational diabetes were examined. The most recent studies included in these reviews, among women with a higher BMI, were further reviewed by the authors to gather more information on their specific use of behaviour-change theories and techniques and their reporting of behavioural and health outcomes ${ }^{(4-7,12,16-19,21,23,24,43-46)}$. Following this, the behavioural science literature was reviewed for more information on behaviour-change theories and techniques $^{(47,48)}$, in addition to published guidelines for the development of lifestyle interventions in non-pregnant populations ${ }^{(26,49)}$. These data were collated and amalgamated among the team. The decision tree was agreed upon through multiple meetings with authors K.M.A., K.L.L. and E.J.O. Thus, we designed a comprehensive tool that can direct researchers to various sources of peer-reviewed information to construct evidence-based antenatal behaviour-change interventions. The procedure for implementing behaviourchange theories and techniques, and the various options for choosing both theories and techniques, are presented in this decision tree (points I-IX) and are based on the work of published authors in the behavioural science field ${ }^{(10,47,48,50)}$. The decision tree was based on MRC guidelines for designing a well-structured, antenatal lifestyle intervention (largely points IX-X and overarching structure of decision tree $)^{(26,49)}$. References are provided throughout the tree.

The MRC guidelines discuss the development of complex interventions in stages, from design through to evaluation $^{(49)}$. Foremost, the design phase of an intervention involves understanding behaviours during pregnancy ${ }^{(47)}$. The corresponding points on the decision tree are: (I) define the health problem of interest, e.g. the impact of excess GWG on maternal and infant health outcomes; (II) identify the specific behaviour(s) that require change, e.g. frequent consumption of high-fat foods; and (III) identify specific target behaviours using 'who, what, when' criteria. The next step is: (IV) to identify barriers and facilitators to adopting new behaviours in a real-world context from the scientific literature. Behaviour-change experts describe this series of steps as key when designing behaviour-change interventions ${ }^{(47)}$. Ideally, conducting an initial qualitative study (V) would identify barriers and facilitators for selecting appropriate behaviour-change 


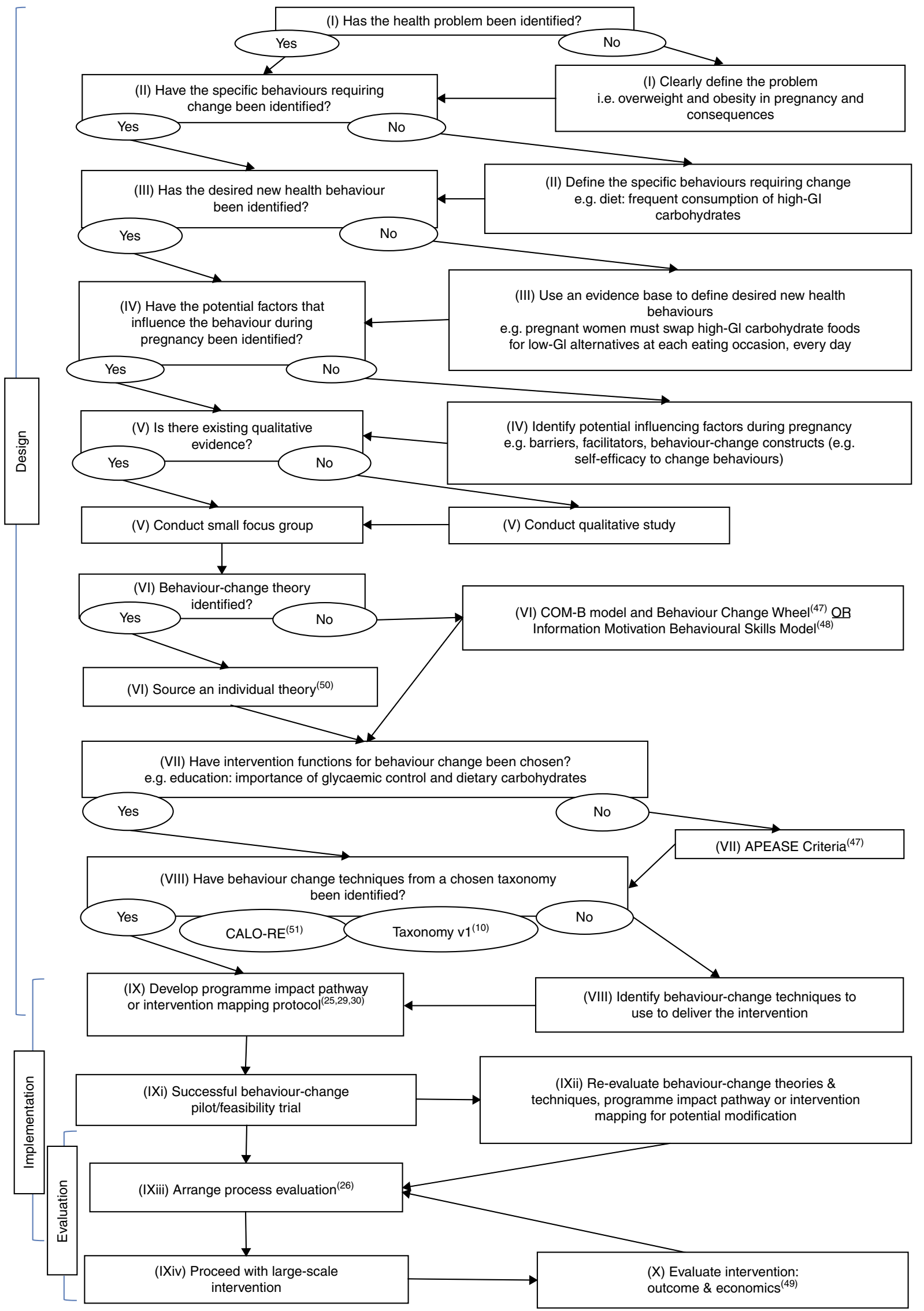

Fig. 1 A decision tree - support for developing behaviour-change interventions in overweight and obese pregnancy (GI, glycaemic index; COM-B, Capability, Opportunity and Motivation to change Behaviour; APEASE, Affordability, Practicality, Effectiveness and cost Effectiveness, Acceptability, Side Effect/Safety, Equity; CAL-ORE, Coventry, Aberdeen and London Refined taxonomy) 
theories and techniques. If a substantial body of qualitative literature already exists to inform the intervention, this may be used to choose behaviour-change theories and techniques. However, in support of MRC guidelines ${ }^{(26,49)}$ we suggest that if an intervention previously described in the literature is to be conducted in a new context (e.g. different countries, ethnicities, age groups), then further qualitative work should be carried out.

The next phase (VI) of intervention design is the selection of behaviour-change theories. Researchers may have identified a behaviour-change theory that supports the intervention a priori; for example, Social Cognitive Theory has been used in pregnancy interventions to encourage behaviour change ${ }^{(21)}$. Alternatively, researchers can select a theory from a comprehensive list of theories $^{(50)}$, or use a general model such as the COM-B ${ }^{(47)}$ or the Information, Motivation Behavioural Skills Model (IMBSM) $^{(48)}$. The COM-B was derived from established theory and encompasses three key behavioural constructs that form the acronym: Capability, Opportunity and Motivation to change Behaviour ${ }^{(47)}$. COM-B eliminates the need for a specific theory to fit an intervention. Similarly, the IMBSM is a generalised model devised on a variation of these constructs: motivation, information and skills to change behaviours. There are guides available on how to use the COM-B and IMBSM as alternatives to specific theories within interventions ${ }^{(47,48)}$.

Once a theory or theoretical basis has been chosen, and target behavioural constructs have been identified, the next step is to: (VII) link behavioural constructs to intervention functions, i.e. the ways in which the intervention is delivered to work $^{(47)}$. For example, if the function of the intervention is to educate or incentivise, researchers can create materials to convey the message of a particular functional component. The COM-B model centres on the Behaviour Change Wheel ${ }^{(47)}$, a tool which maps behavioural constructs to a list of intervention functions such as 'enablement' or 'education'. If using the Behaviour Change Wheel, appropriate intervention functions may be systematically selected using APEASE (Affordability, Practicality, Effectiveness and cost Effectiveness, Acceptability, Side Effect/Safety, Equity) Criteria ${ }^{(47)}$.

Delivery of intervention functions is facilitated through: (VIII) behaviour-change techniques, according to a chosen taxonomy of choice ${ }^{(10,51)}$. Taxonomies are structured lists of behaviour-change techniques to act as a universal language for their reporting ${ }^{(10)}$. This universal language eliminates ambiguity that can arise if techniques are described differently between papers, allowing researchers to compare interventions and conduct metaanalyses $^{(10)}$. At this point, constructing an intervention mapping protocol or devising a PIP (IX) would be useful to hypothesise mechanisms of impact between intervention components, women's behaviours in context and intervention outcomes ${ }^{(25,29)}$. Once ready for implementation, it is strongly suggested that the intervention undergo:
(IXi) pilot/feasibility testing ${ }^{(18,21)}$ to refine behaviourchange theories and techniques and PIP/intervention mapping protocol, if necessary, prior to implementing a larger trial (IXii).

Evaluation is generally the final step when constructing an intervention ${ }^{(26,49)}$. As an exception, process evaluation should run concurrently with the intervention and be performed by a team independent to the researchers conducting the intervention ${ }^{(26)}$ to minimise bias (IXiii). Process evaluation is a type of intervention monitoring that focuses on implementation fidelity and could include the collection of both qualitative and quantitative data. A process evaluation can help determine whether the intervention is delivered appropriately and whether intervention functions work to instigate change ${ }^{(26)}$. In conjunction with intervention mapping, or with the use of a PIP, process evaluation can help establish a mechanism of action between the intervention, behaviours and health outcomes. As proposed by the MRC, planning and conducting a process evaluation ensures the intervention is carefully conceived with respect to its context, the various modes through which it will be implemented and its mechanisms of impact ${ }^{(26)}$. Alternatively, a PIP can be used as a tool to carry out process evaluation, in addition to measuring the mediating effects of an intervention on desired outcomes and the moderating effects of participant characteristics on behavioural outcomes ${ }^{(30)}$. Once process evaluation has been arranged, it is possible to proceed with the full-scale intervention (IXiv).

More traditional intervention evaluation occurs postintervention, and allows researchers to determine whether the intervention: effectively improves diet and physical activity behaviours; is cost-effective; and is sustainable. Thus, the final stage of the decision tree is: $(\mathrm{X})$ evaluate the intervention economics and clinical or behaviourchange outcomes using published guidelines ${ }^{(52,53)}$.

\section{Implications for research and practice}

Using established guidelines ${ }^{(26,27,49)}$ along with the proposed decision tree could help improve the design, implementation, evaluation, reporting and reproducibility of complex behaviour-change interventions in overweight and obese pregnancy.

\section{Adopting behaviour-change theories, techniques and protocols}

Knowledge and understanding of the behaviour-change theories, techniques and protocols, and practical application to one's own academic research or clinical practice is required. We recommend structured training on the use of behaviour-change theories and techniques. Researchers could explore whether workshops at universities in their proximity are accessible. Such training could potentially benefit health and medical science researchers; providing 
knowledge and practical skills to use key behaviourchange theories and techniques.

Adopting evidence-based behaviour-change theories and techniques within clinical practice could improve patient care, in addition to the efficiency and cost-effectiveness of the health-care system ${ }^{(9)}$. While dietitians and psychologists are specifically trained in delivering behaviour-change sessions, not all practitioners are as appropriately equipped with this expertise. Thus, there may be a requirement for clinically based behaviour-change training programmes which have been shown to help health-care professionals to integrate behaviour change within clinical practice ${ }^{(54)}$.

\section{Evaluating behaviour change}

Evaluating the effect of behaviour-change interventions can be challenging, particularly with regard to assessing generalisability ${ }^{(52)}$. Measuring the mediating effects of the intervention on behavioural outcomes and the moderating effects of participant characteristics on behavioural outcomes is important in this regard, in addition to conducting a process evaluation. To our knowledge, only two process evaluations of a diet and physical activity behaviour-change intervention in pregnancy have been published $^{(11,55)}$, reflecting the limited use of process evaluation and perhaps the financial and time constraints involved. Comprehensive outcome and process evaluation of interventions would help guide future behaviourchange research and, subsequently, clinical practice.

\section{Conclusion}

Future implementation of behaviour-change theories and techniques during intervention design is required to build an evidence base for effective intervention components which may be translated into clinical practice. The decision tree proposed in the current paper offers a tool to guide researchers through developing complex behaviour-change antenatal interventions. This tool is likely to be particularly useful to clinicians and health-care professionals conducting research with minimal formal training in qualitative methods. This decision tree provides a concise overview of the process of conducting an intervention and signposts researchers to additional reading that will strengthen their future work.

\section{Acknowledgements}

Financial support: This research received no specific grant from any funding agency in the public, commercial or notfor-profit sectors. Conflict of interest: The authors declare no conflict of interest. Authorship: K.M.A., E.J.O., K.L.L. and F.M.M. conceptualised the manuscript. K.M.A. wrote the manuscript. E.J.O., K.L.L., E.R.G. and F.M.M. critically reviewed and approved the manuscript. Ethics of human subject participation: Not applicable.

\section{References}

1. Nelson SM, Matthews P \& Poston L (2012) Maternal metabolism and obesity: modifiable determinants of pregnancy outcome. Hum Reprod Update 16, 255-275.

2. Phelan S (2010) Pregnancy: a 'teachable moment' for weight control and obesity prevention. Am J Obstet Gynecol 202, $1-16$.

3. Sui Z, Turnbull D \& Dodd J (2013) Enablers of and barriers to making healthy change during pregnancy in overweight and obese women. Australas Med J 6, 565-577.

4. Phelan S, Phipps MG, Abrams B et al. (2011) Randomized trial of a behavioral intervention to prevent excessive gestational weight gain: the Fit for Delivery Study. Am J Clin Nutr 93, 772-779.

5. Quinlivan JA, Lam LT \& Fisher J (2011) A randomised trial of a four-step multidisciplinary approach to the antenatal care of obese pregnant women. Aust NZ J Obstet Gynaecol 51, $141-146$

6. Vítolo MR, Bueno MSF \& Gama CM (2011) Impact of a dietary counseling program on the gain weight speed of pregnant women attended in a primary care service. Rev Bras Ginecol Obstet 33, 13-19.

7. Wolff S, Legarth J, Vangsgaard K et al. (2008) A randomized trial of the effects of dietary counseling on gestational weight gain and glucose metabolism in obese pregnant women. Int J Obes (Lond) 32, 495-501.

8. Guelinckx I, Devlieger R, Mullie P et al. (2010) Effect of lifestyle intervention on dietary habits, physical activity, and gestational weight gain in obese pregnant women: a randomized controlled trial. Am J Clin Nutr 91, 373-380.

9. Birdsall KM, Vyas S, Khazaezadeh N et al. (2009) Maternal obesity: a review of interventions. Int J Clin Pract 63, 494-507.

10. Michie S, Richardson M, Johnston M et al. (2013) The behavior change technique taxonomy (v1) of 93 hierarchically clustered techniques: building an international consensus for the reporting of behavior change interventions. Ann Behav Med 46, 81-95.

11. Poston L, Briley AL, Barr S et al. (2013) Developing a complex intervention for diet and activity behaviour change in obese pregnant women (the UPBEAT trial); assessment of behavioural change and process evaluation in a pilot randomised controlled trial. BMC Pregnancy Childbirth 13, 148.

12. Rhodes ET, Pawlak DB, Takoudes TC et al. (2010) Effects of a low-glycemic load diet in overweight and obese pregnant women: a pilot randomized controlled trial. Am J Clin Nutr 92, 1306-1315.

13. Goodrich K, Cregger M, Wilcox S et al. (2013) A qualitative study of factors affecting pregnancy weight gain in African American women. Matern Child Health J 17, 432-440.

14. Thomas M, Vieten C, Adler N et al. (2014) Potential for a stress-reduction intervention to promote healthy gestational weight gain: focus groups with low income pregnant women. Womens Health Issues 24, Suppl. 3, e305-e311.

15. McLeroy KR, Bibeau D, Steckler A et al. (1988) An ecological perspective on health promotion programs. Health Educ Q 15, 351-377.

16. Ong MJ, Guelfi KJ, Hunter T et al. (2009) Supervised homebased exercise may attenuate the decline of glucose tolerance in obese pregnant women. Diabetes Metab 35, 418-421.

17. Thornton YS, Smarkola C, Kopacz SM et al. (2009) Perinatal outcomes in nutritionally monitored obese pregnant women: a randomized clinical trial. I Natl Med Assoc 101, 569-577.

18. Nascimento SL, Surita FG, Parpinelli M et al. (2011) The effect of an antenatal physical exercise programme on maternal/perinatal outcomes and quality of life in overweight and obese pregnant women: a randomised clinical trial. BJOG 118, 1455-1463. 
19. Vinter CA, Jensen DM, Ovesen P et al. (2011) The LiP (Lifestyle in Pregnancy) study: a randomized controlled trial of lifestyle intervention in 360 obese pregnant women. Diabetes Care 34, 2502-2507.

20. Dodd JM, Turnbull D, McPhee AJ et al. (2014) Antenatal lifestyle advice for women who are overweight or obese: LIMIT randomised trial. BMJ 348, g1285.

21. Poston L, Bell R, Croker H et al. (2015) Effect of a behavioural intervention in obese pregnant women (the UPBEAT study): a multicentre, randomised controlled trial. Lancet Diabetes Endocrinol 3, 767-777.

22. John E, Cassidy DM, Playle R et al. (2014) Healthy eating and lifestyle in pregnancy (HELP): a protocol for a cluster randomised trial to evaluate the effectiveness of a weight management intervention in pregnancy. BMC Public Health 14, 439.

23. Dekker Nitert M, Barrett HL, Denny KJ et al. (2015) Exercise in pregnancy does not alter gestational weight gain, MCP-1 or leptin in obese women. Aust N Z J Obstet Gynaecol 55 , 27-33.

24. Dodd JM, Newman A, Moran LJ et al. (2014) The effect of antenatal dietary and lifestyle advice for women who are overweight or obese on maternal diet and physical activity: the LIMIT randomized trial. BMC Med 12, 161.

25. Bartholemew Eldredge LK, Markham CM, Ruiter RAC et al. (2016) Planning Health Promotion Programs: An Intervention Mapping Approach, 4th ed. San Francisco, CA: Jossey-Bass.

26. Moore GF, Audrey S, Barker M et al. (2015) Process evaluation of complex interventions: Medical Research Council guidance. BMJ 350, h1258.

27. National Institute for Health and Clinical Excellence (2007) Behaviour Change at Population, Community and Individual Levels. NICE Public Health Guidance no. 6. London: NICE.

28. Collins LM, Nahum-Shani I \& Almirall D (2014) Optimization of behavioral dynamic treatment regimens based on the sequential, multiple assignment, randomized trial (SMART). Clin Trials 11, 426-434.

29. Avula R, Menon P, Saha KK et al. (2013) A program impact pathway analysis identifies critical steps in the implementation and utilization of a behavior change communication intervention promoting infant and child feeding practices in Bangladesh. J Nutr 143, 2029-2037.

30. Mbuya MNN, Jones AD, Ntozini R et al. (2015) Theorydriven process evaluation of the SHINE trial using a program impact pathway approach. Clin Infect Dis 61, Suppl. 7, S752-S758.

31. Tanentsapf I, Heitmann BL \& Adegboye AR a (2011) Systematic review of clinical trials on dietary interventions to prevent excessive weight gain during pregnancy among normal weight, overweight and obese women. BMC Pregnancy Childbirth 11, 81.

32. Thangaratinam S, Rogozinska E, Jolly K et al. (2012) Effects of interventions in pregnancy on maternal weight and obstetric outcomes: meta-analysis of randomised evidence. BMJ 344, e2088.

33. Gardner B, Wardle J, Poston L et al. (2011) Changing diet and physical activity to reduce gestational weight gain: a meta-analysis. Obes Rev 12, e602-e620.

34. Brown MJ, Sinclair M, Liddle D et al. (2012) A systematic review investigating healthy lifestyle interventions incorporating goal setting strategies for preventing excess gestational weight gain. PLoS One 7, e39503.

35. Skouteris H, Hartley-Clark L, McCabe M et al. (2010) Preventing excessive gestational weight gain: a systematic review of interventions. Obes Rev 11, 757-768.

36. Streuling I, Beyerlein A \& von Kries R (2010) Can gestational weight gain be modified by increasing physical activity and diet counseling? A meta-analysis of interventional trials. Am J Clin Nutr 92, 678-687.
37. Oteng-Ntim E, Varma R, Croker H et al. (2012) Lifestyle interventions for overweight and obese pregnant women to improve pregnancy outcome: systematic review and metaanalysis. BMC Med 10, 47.

38. Choi J, Fukuoka Y \& Lee JH (2013) The effects of physical activity and physical activity plus diet interventions on body weight in overweight or obese women who are pregnant or in postpartum: a systematic review and meta-analysis of randomized controlled trials. Prev Med 56, 351-364.

39. Dodd JM, Grivell RM, Crowther CA et al. (2010) Antenatal interventions for overweight or obese pregnant women: a systematic review of randomised trials. BJOG 117, 1316-1326.

40. Agha M, Agha RA \& Sandell J (2014) Interventions to reduce and prevent obesity in pre-conceptual and pregnant women: a systematic review and meta-analysis. PLoS One 9, e95132.

41. Bain E, Crane M, Tieu J et al. (2015) Diet and exercise interventions for preventing gestational diabetes mellitus. Cochrane Database Syst Rev issue 4, CD010443.

42. Tieu J, Shepherd E, Middleton P et al. (2013) Dietary advice in pregnancy for preventing gestational diabetes mellitus a Cochrane review. J Paediatr Child Health 49, 118-119.

43. Callaway LK, Colditz PB, Byrne NM et al. (2010) Prevention of gestational diabetes: feasibility issues for an exercise intervention in obese pregnant women. Diabetes Care 33, 1457-1459.

44. Oostdam N, Van Poppel MNM, Wouters MG a J et al. (2012) No effect of the FitFor2 exercise programme on blood glucose, insulin sensitivity, and birthweight in pregnant women who were overweight and at risk for gestational diabetes: results of a randomised controlled trial. BJOG 119, 1098-1107.

45. Hui AL, Ludwig SM, Gardiner P et al. (2006) Communitybased exercise and dietary intervention during pregnancy: a pilot study. Can J Diabetes 30, 1-7.

46. Renault KM, Nørgaard K, Nilas L et al. (2014) The Treatment of Obese Pregnant Women (TOP) study: a randomized controlled trial of the effect of physical activity intervention assessed by pedometer with or without dietary intervention in obese pregnant women. Am J Obstet Gynecol 210, 134.e1-e9.

47. Michie S, Atkins L \& West R (2014) The Behaviour Change Wheel: A Guide to Designing Interventions. London: Silverback Publishing.

48. Fisher WA, Fisher JD \& Harman J (1992) The InformationMotivation-Behavioural Skills Model: a general social psychological approach to understanding and promoting health behavior. In Social Psychological Foundations of Health and Illness, pp. 82-106 [J Suls and KA Wallston, editors]. Oxford: Blackwell Publishing.

49. Craig P, Dieppe P, Macintyre S et al. (2008) Developing and evaluating complex interventions: the new Medical Research Council guidance. BMJ 337, a1655.

50. Michie S, West R, Campbell R et al. (2014) ABC of Behaviour Change Theories: An Essential Resource for Researchers, Policy Makers and Practitioners. London: Silverback Publishing.

51. Michie S, Ashford S, Sniehotta FF et al. (2011) A refined taxonomy of behaviour change techniques to help people change their physical activity and healthy eating behaviours: the CALO-RE taxonomy. Psychol Health 26, 1479-1498.

52. Denford S, Abraham C, Smith J et al. (2015) Designing and evaluating behavior change interventions to promote health. In The Psychology of Change: Life Contexts, Experiences, and Identities, pp. 151-170 [KJ Reynolds and NR Branscombe, editors]. New York: Psychology Press.

53. Glasgow RE, Bull SS, Gillette C et al. (2002) Behavior change intervention research in healthcare settings - a review of recent reports with emphasis on external validity. Am J Prev Med 23, 62-69.

54. Mostofian F, Ruban C, Simunovic N et al. (2015) Changing physician behavior: what works? Am J Manag Care 21, 75-84.

55. Simpson SA, Cassidy D, John E et al. (2014) 'Healthy Eating and Lifestyle in Pregnancy (HELP)' trial: process evaluation framework. Pregnancy Hypertens 4, 233. 